OPEN ACCESS

Edited by:

Seth Corey,

Cleveland Clinic, United States

Reviewed by:

Quen Mok,

Great Ormond Street Hospital for

Children NHS Foundation Trust,

United Kingdom

Tanıl Kendirli,

Ankara University, Turkey

*Correspondence:

Yimei Ma

mayimei90@163.com

†These authors have contributed equally to this work

Specialty section:

This article was submitted to

Pediatric Hematology and Hematological Malignancies,

a section of the journal

Frontiers in Pediatrics

Received: 14 October 2021

Accepted: 07 January 2022

Published: 03 March 2022

Citation:

Deng Z, Qin J, Sun H, Xv F and Ma Y

(2022) Effectiveness of Impregnated

Central Venous Catheters on

Catheter-Related Bloodstream

Infection in Pediatrics.

Front. Pediatr. 10:795019

doi: 10.3389/fped.2022.795019

\section{Effectiveness of Impregnated Central Venous Catheters on Catheter-Related Bloodstream Infection in Pediatrics}

\author{
Zhengrong Deng ${ }^{1,2+}$, Jiangwei Qin ${ }^{3 \dagger}$, Huanbin Sun ${ }^{4}$, Furong $X v^{5}$ and Yimei Ma ${ }^{1,2 *}$ \\ ${ }^{1}$ Department of Pediatrics, West China Second University Hospital, Sichuan University, Chengdu, China, ${ }^{2}$ Key Laboratory of \\ Birth Defects and Related Diseases of Women and Children (Sichuan University), Ministry of Education, Chengdu, China, \\ ${ }^{3}$ West China Hospital, Sichuan University, Chengdu, China, ${ }^{4}$ West China School of Basic Medical Sciences and Forensic \\ Medicine, Sichuan University, Chengdu, China, ${ }^{5}$ Wuyuzhang Honors College, Sichuan University, Chengdu, China
}

Background: The efficacy and safety of impregnated central venous catheters (CVCs) in pediatrics remain controversial. The purpose of this study was to evaluate the efficacy of impregnations for the prevention of catheter-related bloodstream infection (CRBSI).

Methods: We searched the following five electronic databases: Medline, PubMed, Cochrane, Embase, and the Web of Science for randomized controlled trials (RCTs) up to March 2021. Pooled risk ratios (RRs) with 95\% confidence intervals (Cls) were calculated using a fixed-effects model. Assessment of publication biases was evaluated by Egger's test. Heterogeneity between studies was assessed based on the chi-square test and $l^{2}$ statistics, and sensitivity analysis and subgroup analysis were also performed.

Results: A total of six RCTs with 3,091 patients were included. Impregnated CVCs provided significant benefits in reducing the risk of CRBSI (RR $=0.41,95 \% \mathrm{Cl}$ : $0.26-0.66)$ in pediatric patients, especially in the pediatric group. No publication bias was observed in the Egger test for the risk of CRBSI. Drug type is a source of heterogeneity.

Conclusion: Antimicrobial-impregnated CVCs are beneficial to prevent CVC-related complications in pediatrics.

Keywords: antibiotics, impregnations, central venous catheters, bloodstream infection, pediatrics

\section{INTRODUCTION}

Central venous catheters (CVCs) are clinically important in the treatment of venous pressure monitoring, infusion of drugs, and nutrient fluid supplementation (1). However, the widespread use of CVCs has been shown to increase the risk of developing catheter-related bloodstream infection (CRBSI) (2). It has been reported that CRBSIs can occur in 13-20\% of catheterized newborns by the Centers for Disease Control and Prevention (3) and that 1 in 10 children with a central venous catheter develops a central line-associated bloodstream infection (4). It is wellknown that CRBSIs increase children's mortality, exposure to antibiotics, direct cost, and length of stay in the hospital $(5,6)$. 
The prevention strategies of CRBSI being recommended include highlighting hand hygiene (7), maximal sterile barrier precautions (8), and avoidance of the femoral site for catheter insertion (9). However, with each strategy having potential clinical advantages, it is unclear whether there are better clinical outcomes than the other treatment regimens. The most promising approach is the use of impregnated CVCs, which is recommended by the US and UK national guidelines for patients at high risks of infection (10). Impregnated CVC effectiveness comparing standard, non-impregnated CVCs has been provided in previous adult systematic analyses (11-13). Antimicrobial agents such as chlorhexidine-, minocycline-rifampicin-, and silver sulfadiazine-impregnated (14) CVCs have shown beneficial outcomes in preventing CRBSI in adults. Impregnated central venous catheters, however, have not enough evidence for their effect on reducing the rates of CRBSI in pediatrics. Furthermore, several new RCTs on this issue have been reported.

Given this background, we performed a meta-analysis study of available studies to evaluate the efficacy of central venous catheters impregnated with heparin and antimicrobial agents in comparison with standard and non-impregnated catheters on the prevention of CRBSI.

\section{MATERIALS AND METHODS}

We conducted the meta-analysis according to the Preferred Reporting Items for Systematic Review and Meta-Analysis Protocol (PRISMAP) (15).

\section{Search Strategy and Selection Criteria}

The search was performed up to March 2021 in PubMed, Medline, Embase, the Cochrane Central Register of Controlled Trials, and the Web of Science using MeSH terms and text words for published clinical trials, and the references of relevant meta-analyses and other internet sources for unpublished clinical trials were also considered. The following terms were adjusted according to different search rules in each database: "impregnated," "catheters," "pediatrics," "bloodstream," and "infections." We also searched ClinicalTrials.gov and the WHO International Clinical Trials Registry Platform for unpublished, planned, or ongoing trial reports. In addition, we contacted the authors of the included RCTs to obtain additional data if necessary. All studies were imported into EndNote for duplication exclusion.

\section{Criteria for Included Studies}

All potential studies were screened based on the PICOS (population, intervention, comparator, outcome, and study design) standard. We defined the terms as follows: (1) Population: all patients with vascular catheters inserted for an expected duration of more than $72 \mathrm{~h}$ under the age of 18 ; (2) Intervention: the intervention method was impregnation-treated CVCs with no limitation of drug types, and the control group refers to conventional, non-impregnated CVCs; (3) Comparison: the objective was to compare the efficacy of impregnated CVCs and non-impregnated CVCs in the prevention of CRBSI in children; (4) Outcomes: the outcomes included the incidence of
CRBSIs and thrombosis; (5) Study design: all study designs were prospective RCTs.

\section{Outcome Measures}

The primary outcome in this meta-analysis was the incidence of CRBSIs. Furthermore, we also considered the incidence of thrombosis as the secondary outcome with incomplete data among the included studies.

\section{Data Extraction}

Two reviewers (Zhengrong Deng and Jiangwei Qin) independently evaluated the titles, abstracts, and full texts of identified studies using predefined criteria and extracted study characteristics and outcome data for each study, such as first author name, publication year, country, age of population, numbers of participants, interventions, clinical signs, and brief conclusion, with all the data cross-checked by other authors. Any disagreements were resolved to a consensus by team discussion.

\section{Quality Assessment}

The risk of bias of the included RCTs was evaluated using the Cochrane Risk of Bias Scale independently using the following criteria: (1) random sequence generation (selection bias), (2) allocation concealment (selection bias), (3) blinding of participants and personnel (performance bias), (4) incomplete outcome data (attention bias), (5) selective reporting (reporting bias), and (6) other sources of bias. For each criterion, studies were classified as having a low, high, or unclear risk of bias.

\section{Statistical Analyses}

The analyses were performed in Review Manager (Version 5.3) and $\mathrm{R}$ (Version 4.04). The incidence of CRBSI and thrombosis are binary outcomes, so risk ratios (RRs) with $95 \%$ confidence intervals (95\% CIs) were calculated using the Mantel-Haenszel method. If there was no statistically significant heterogeneity among the studies $\left(I^{2}<50 \%, p>0.05\right)$, a fixed-effects model was used; otherwise, a random-effects model was considered. We set $p<0.05$ as statistically significant for hypothesis testing. Heterogeneity between studies was also assessed based on the chi-square test by $I^{2}$ statistics. Assessment of publication biases was evaluated by Egger regression. Sensitivity analysis was conducted to identify the influence of each study on the synthesized results. $p<0.05$ was considered statistically significant. Subgroup analyses based on impregnation drug type and between neonates (age $\leq 28$ days) and children (28 days $<$ age $\leq 18$ years) were undertaken to determine the source of heterogeneity. Furthermore, meta-regression analyses were used to detect whether other variables, such as publication year and bloodstream infection (BSI) reported, caused heterogeneity to some extent.

\section{RESULTS}

\section{Study Selection and Data Extraction}

The search identified 813 records, of which 229 duplicates were removed. In addition, 18 other studies were included from the reference list of a relevant review and other sources. Of the 


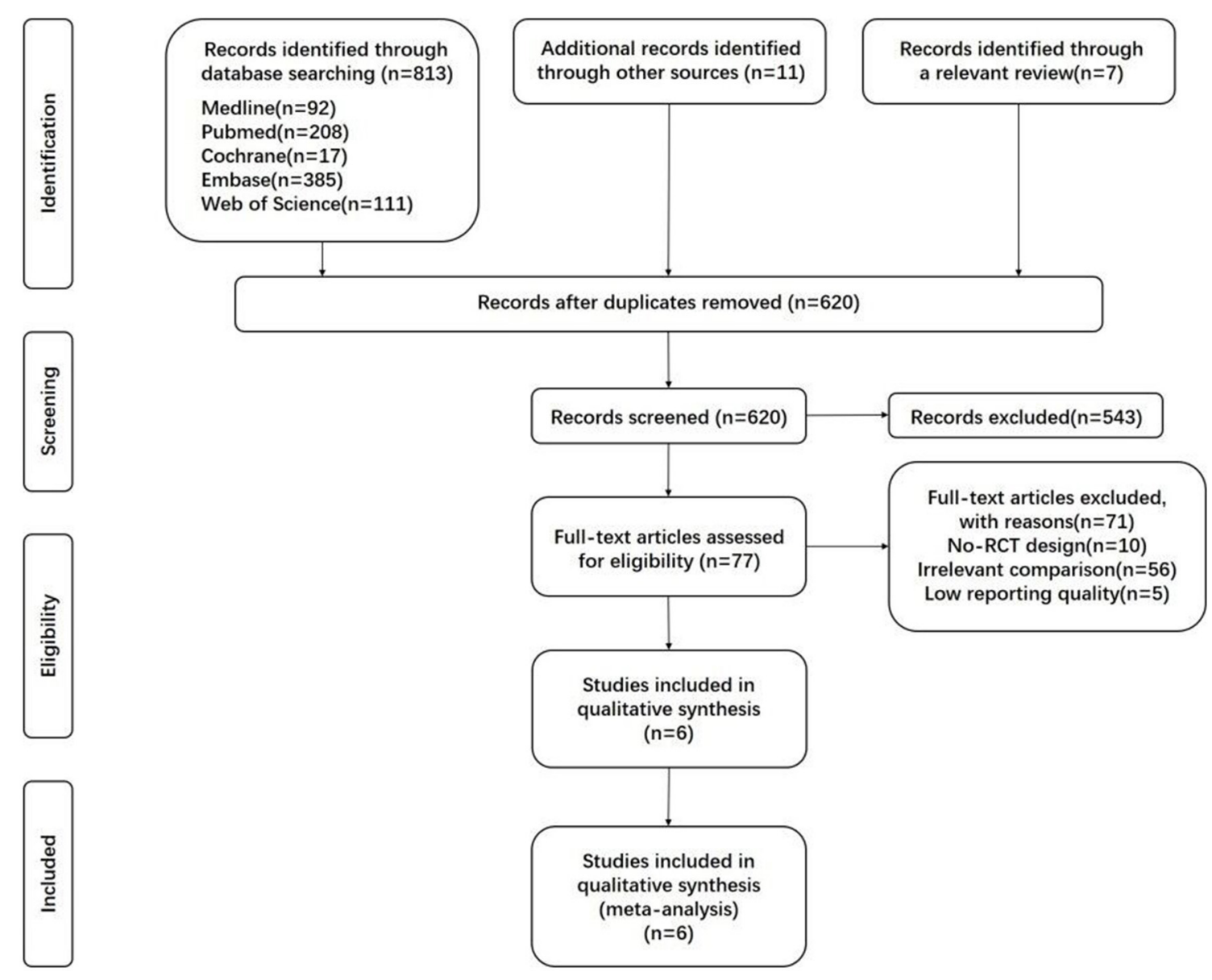

FIGURE 1 | PRISMA flowchart for study selection.

remaining 620 articles, 77 were relevant after inspecting the titles. During the full-text screening, 71 articles were excluded due to No-RCT design, irrelevant comparison, or low reporting quality, leaving six articles to be included in this meta-analysis. These studies included a total of 3,091 patients, with 1,781 patients for impregnated CVC intervention and 1,310 patients for standard CVC intervention. Figure 1 presents the PRISMA flowchart for study selection. Supplementary Table S1 (16-21) presents the characteristics of each study. For the type of CVC, other details were reported among the included RCTs, as described in Supplementary Table S2. There would be little probability that heterogeneity originated from catheter materials because they are all composed of polyurethane. Conclusions suggested that three studies detected that antimicrobial-impregnated CVC did not provide a protective effect of CRBSI, while three other studies found that antimicrobial-impregnated CVC significantly reduced the risk of CRBSI.

\section{Bias Evaluation}

The results of the quality assessment of the five RCTs included are presented in Figure 2. The outcomes indicated the risks of bias for each included study. Briefly, all included RCTs mentioned randomization in their reports, but only two RCTs reported the methods to generate random sequences. Two studies failed to report the methods to perform allocation concealment. Four studies were rated as having a high risk of performance bias, as they were unable to blind the personnel or participants to the intervention allocated. One RCTs reported a blind design during the outcome assessment. No other kinds of biases were found.

\section{Primary Outcome}

\section{The Rate of CRBSIs}

The impregnated CVCs had lower rates than the control group in the risk of CRBSI $(\mathrm{RR}=0.41 ; 95 \%$ CI 0.26 to $0.66 ; p=0.0002$, weighted based on sample size). A fixed effects model was used for the meta-analysis because of moderate heterogeneity $\left(I^{2}=\right.$ $45 \%, p=0.11$ ) (Figure 3A). We also obtained $Q=9.04 . I^{2}$ and $Q$ suggest the appearance of heterogeneity amounting equally with random error.

\section{Publication Bias}

We performed Egger's test of funnel plot asymmetry on the risk of CRBSI in Figure 3B, which indicated that no publication bias was detected $(t=-1.05, p=0.3520>0.05)$. 


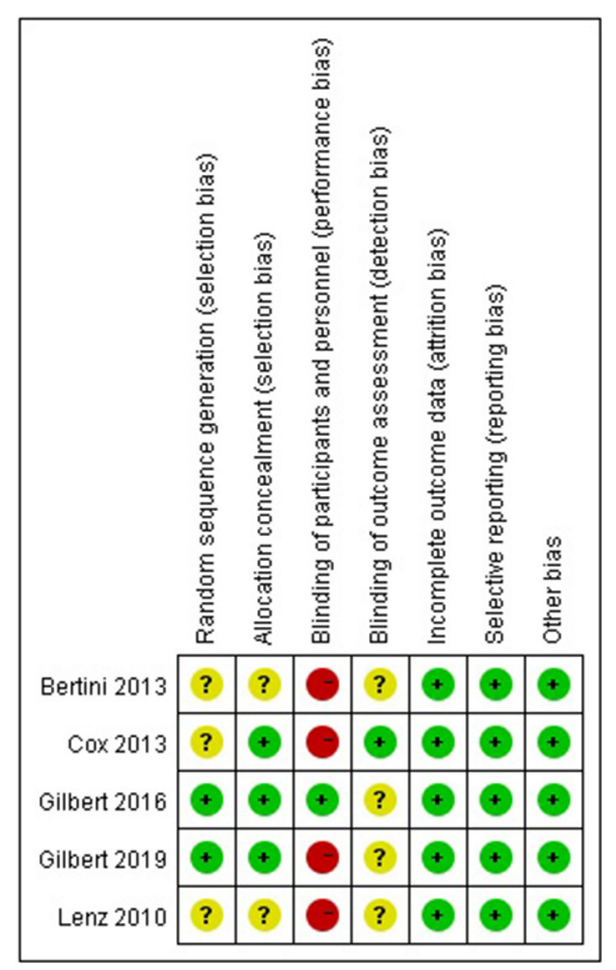

FIGURE 2 | Risk of bias summary.

\section{Sensitivity Analyses}

Considering the moderate heterogeneity in the synthetic result, we conducted a sensitivity analysis by excluding 1 study at a time to estimate whether the results could have been affected markedly by a single study (Figure $3 \mathrm{C}$ ). The results showed that Pierce et al. (16) accounted for a significant percentage of the bias of effect size. However, we found that the overall results were not changed by exclusion of any included RCTs. Considering that Pierce et al. (16) is one of only two studies using heparin-bonded CVCs, we suspected that catheter type is a potential source of heterogeneity. Heparin has been recognized as a valuable antithrombotic drug without direct antibacterial effects compared with chlorhexidine antiseptics and silver ions (22). Pierce et al. (16) suggested that heparin may prevent CRBSI by reducing thrombosis and bacterial colonization, acting as an indirect action on CRBSI.

\section{Subgroup Analysis}

To test our assumption, we conducted subgroup analysis stratified by heparin-impregnated CVCs and antimicrobialimpregnated catheters. As Figure 4 shows, the antimicrobialimpregnated CVC group presented lower heterogeneity $\left(I^{2}=\right.$ $26 \%, p=0.25)$, while impregnated heparin showed higher heterogeneity $\left(I^{2}=84 \%, p=0.01\right)$, indicating that the efficiency of heparin impregnated on CRBSI was unstable. In addition, it showed that the antimicrobial-impregnated catheter provided more benefits ( $\mathrm{RR}=0.48$; $95 \% \mathrm{CI}$ : $0.27-0.86$, weighted based on sample size) in reducing the risk of CRBSI among the included RCTs compared to the heparin-impregnated CVCs.

We also added an exploratory subgroup analysis regarding the differences in infection rates between neonates (age $\leq 28$ days) and children ( 28 days $<$ age $\leq 18$ years) for discrepant immune system development. The results shown in Figure 5 indicate that both groups had a relatively large scale of heterogeneity $\left(I^{2}=\right.$ $51 \%, p=0.11$ in the neonate group; $I^{2}=66 \%, p=0.09$ in the children group). It should be noted that although results with heterogeneity should be considered conservatively, impregnated CVCs seemed to protect children from CRBSI with an $\mathrm{RR}=0.37$ (95\% CI: 0.21-0.68, weighted based on sample size).

\section{Secondary Outcome}

\section{The Rate of Thrombosis}

A meta-analysis of three studies revealed no significant differences in the rate of thrombosis between impregnated and standard CVCs $(16,19,20)(\mathrm{RR}=0.90,95 \% \mathrm{CI}: 0.74-1.08, p$ $=0.253)$. A fixed model was used because no heterogeneity was noted among the studies $\left(I^{2}=14 \%, p=0.31\right)$. The results are presented in Figure 6.

\section{DISCUSSION}

We exhaustively collected RCTs related to impregnated CVCs in pediatrics, which included agents such as heparin, AgION, minocycline, miconazole, and rifampicin. Heparin is a natural anticoagulant substance in animals, and its anti-infective function was suspected to be related to preventing bacterial colonization through beneficial hemodynamic function. However, as we described in the subgroup analysis in Figure 4, the clinical anti-infective effect is unstable and requires other studies for more convincing results. AgION, as a patented silver compound, has been reported to be effective in adult treatment (23) because of the bactericidal activity of silver ions. Minocycline, a second-generation, semisynthetic tetracycline, can effectively reduce the risk of CRBSI in pediatrics due to its antibiotic properties against both Gram-positive and Gramnegative bacteria (20). Miconazole and rifampicin combination is a common antimicrobial combination impregnated strategy of catheters, which was estimated to reduce infections from 5 to $0 \%$ to save $\$ 500,000$ per 850 catheters used (24). The use of the above antimicrobial agents can theoretically reduce the risk of relevant infections, as reported in our results.

It has been reported that pediatric intensive care units (PICUs) have one of the highest rates of acquired bloodstream infections in hospitals, especially after cardiac surgery (25), with central venous catheters being a frequent source. PICU admission for patients with nosocomial primary BSI would have $\sim 6$ times the direct cost compared with patients without BSI, as reported by St Louis Children's Hospital in 2005 (26).

To date, much new evidence has basically unified the perspective that impregnated CVCs are recommended for adults to reduce bloodstream infections. Chong et al. conducted a network meta-analysis including 60 studies with 17,255 catheters and investigated the effects of 14 impregnation drugs on CRBSI and catheter colonization. Significant CRBSI 


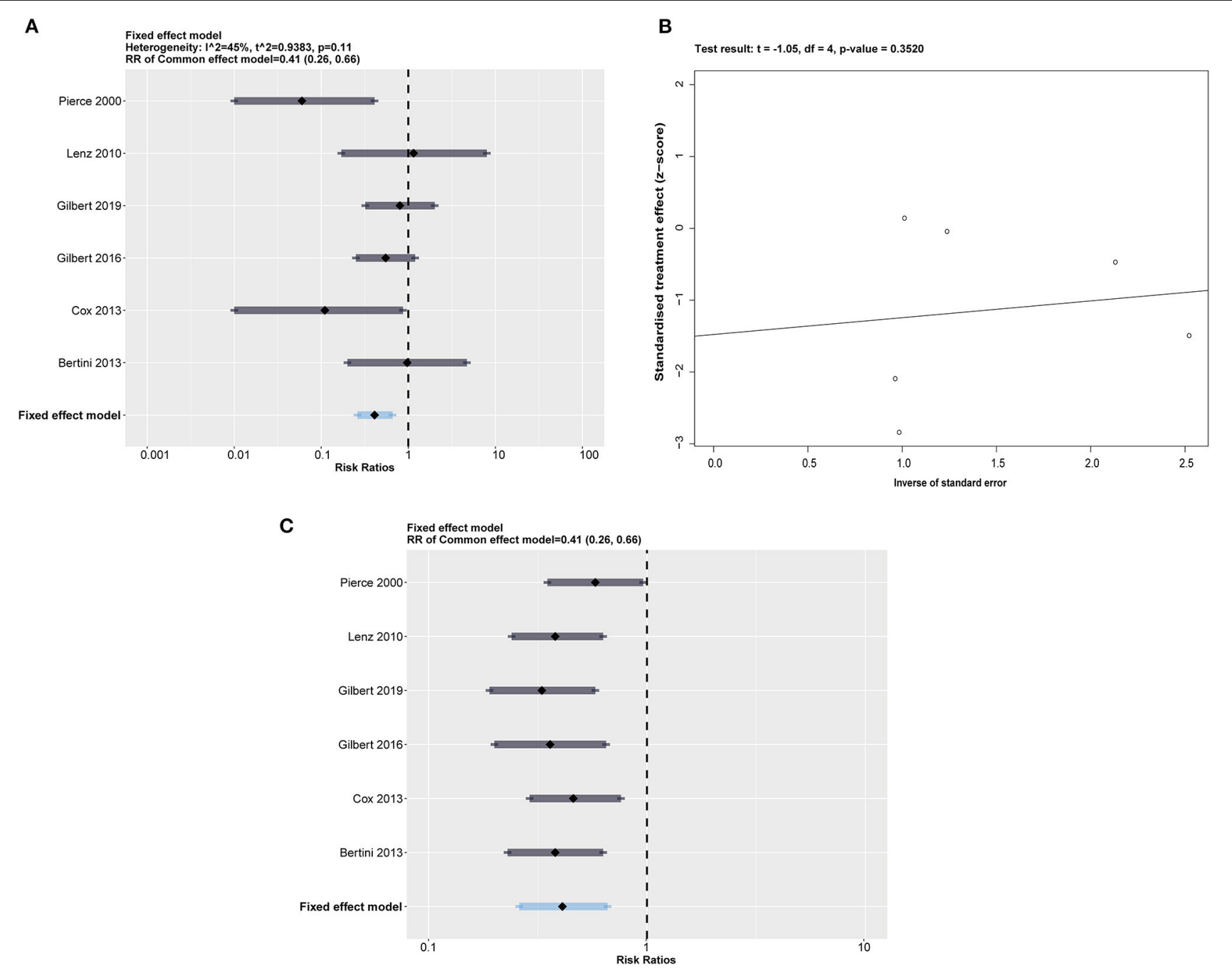

FIGURE 3 | (A) Forest plot of the risk of CRBSI for impregnated catheters. All impregnated medicines were calculated together, with a summary RR $=0.41(0.26$, 0.66) under a fixed effect model. (B) Egger's test plot shows an acceptable $p>0.3520$. (C) The sensitivity analysis performed by eliminating one included study in a time represents a relatively robust result.

reduction was associated with minocycline-rifampicin $(\mathrm{RR}=$ 0.29 [95\% CI: 0.16-0.52]) and silver ( $\mathrm{RR}=0.57$ [95\% CI: 0.38-0.86]) impregnations compared to no impregnation. For colonization, miconazole-rifampicin ( $\mathrm{RR}=0.14$ [95\% CI: 0.050.36]), 5-fluorouracil ( $\mathrm{RR}=0.34$ [95\% CI: 0.14-0.82]), and chlorhexidine-silver sulfadiazine $(\mathrm{RR}=0.60$ [95\% CI: 0.50-0.72]) impregnations showed significant decreases compared with nonimpregnation (11). Wei et al. (12) reported that chlorhexidineimpregnated dressings provided significant benefits in reducing the risk of catheter colonization ( $\mathrm{OR}=0.46,95 \% \mathrm{CI}$ : $0.36-$ 0.58 ) and CRBSI (OR $=0.60,95 \% \mathrm{CI}: 0.42-0.85)$. Huang et al. (27) analyzed chlorhexidine-, heparin-, and antimicrobialimpregnated CVCs in a systematic review and gave a positive conclusion. All of the above meta-analyses indicate positive outcomes on impregnations, including minocycline, rifampicin, silver ion, chlorhexidine, and heparin, in the control of CRBSI in adults. In pediatrics, however, there are no such large simple size systematic analyses to prove whether impregnated CVCs are effective or not due to a lack of enough RCTs. It is necessary to combine existing studies related to children to complement clinical guidelines.

Our systematic review and meta-analysis provided the most recent and comprehensive analysis of the efficiency of impregnated CVCs for the prevention of CRBSI in pediatrics. With six RCTs included, the results of this meta-analysis suggest that the use of impregnated CVCs is beneficial to reduce the risk of CRBSI for patients with CVC and is an effective anti-infection strategy in preventing CRBSI $(\mathrm{RR}=0.41 ; 95 \% \mathrm{CI}$ : $0.26-0.66$; $p=0.0002$ ). Our further subgroup analysis indicated that the use of antimicrobial drug-impregnated CVCs had a significantly lower risk of CRBSI $(\mathrm{RR}=0.48)$ than the conventional catheter group in the pediatric population. In addition, after considering 


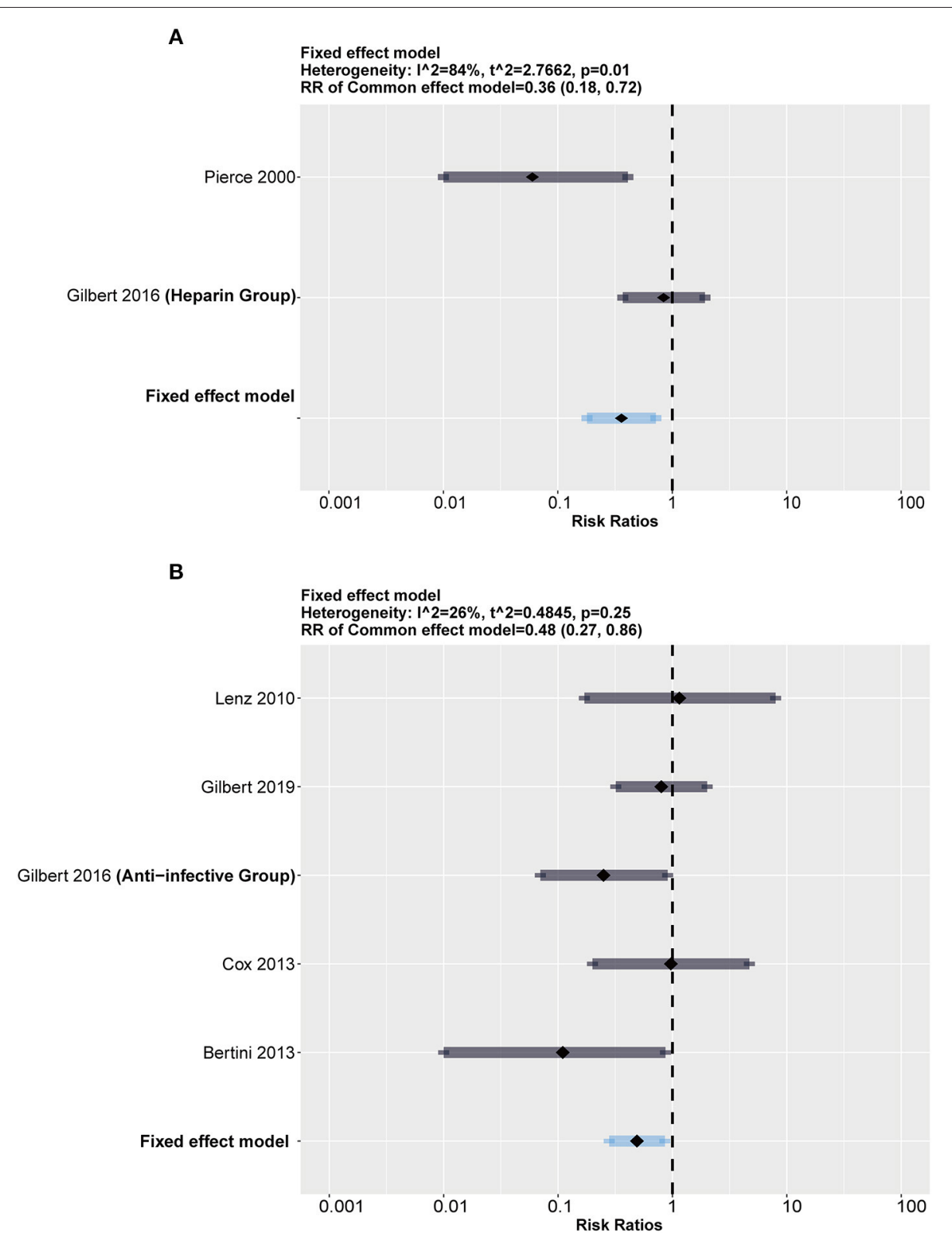

FIGURE 4 | Subgroup analysis between heparin and antimicrobial drugs. The forest showed a significant difference in both groups but a much higher heterogeneity in the heparin group than in the antimicrobial group. (A) Forest plot in heparin group (compared with standard). (B) Forest plot in antimicrobial group (compared with standard).

differences between neonates and children, the age cutoff at 28 days supported a prominent prevention effect in the pediatric group ( 28 days $<$ age $\leq 18$ years). Our results support the benefits antimicrobial drug-impregnated CVCs would have in pediatric patients, especially in children.

The results were consistent with previous findings (28), but with more RCTs included for synthesized analysis, our results do provide more strength in increasing the statistical effectiveness. The newly included study by Gilbert et al. (21) represented a better quality in both random sequence generation and allocation concealment, also with a higher weight for larger sample sizes. Its risk ratio $(\mathrm{RR}=0.80,95 \% \mathrm{CI}: 0.31-2.01)$ exceeded the combined risk ratio in Wu et al. (28) ( $\mathrm{RR}=0.28,95 \% \mathrm{CI}: 0.13$ and 1.09$)$, but this RCT with high quality led to a smaller confidence interval and a beneficial effect of impregnated CVCs.

There are several limitations that need to be considered in this meta-analysis. First, the main limitation is that our meta-analysis only included six eligible RCTs, which were relatively small in the establishment of prognostic value. Second, the antimicrobial concentration used for impregnated CVC is uncertain. Third, we only performed subgroup analyses stratified by impregnation type and age population but not by the frequency of dressing 

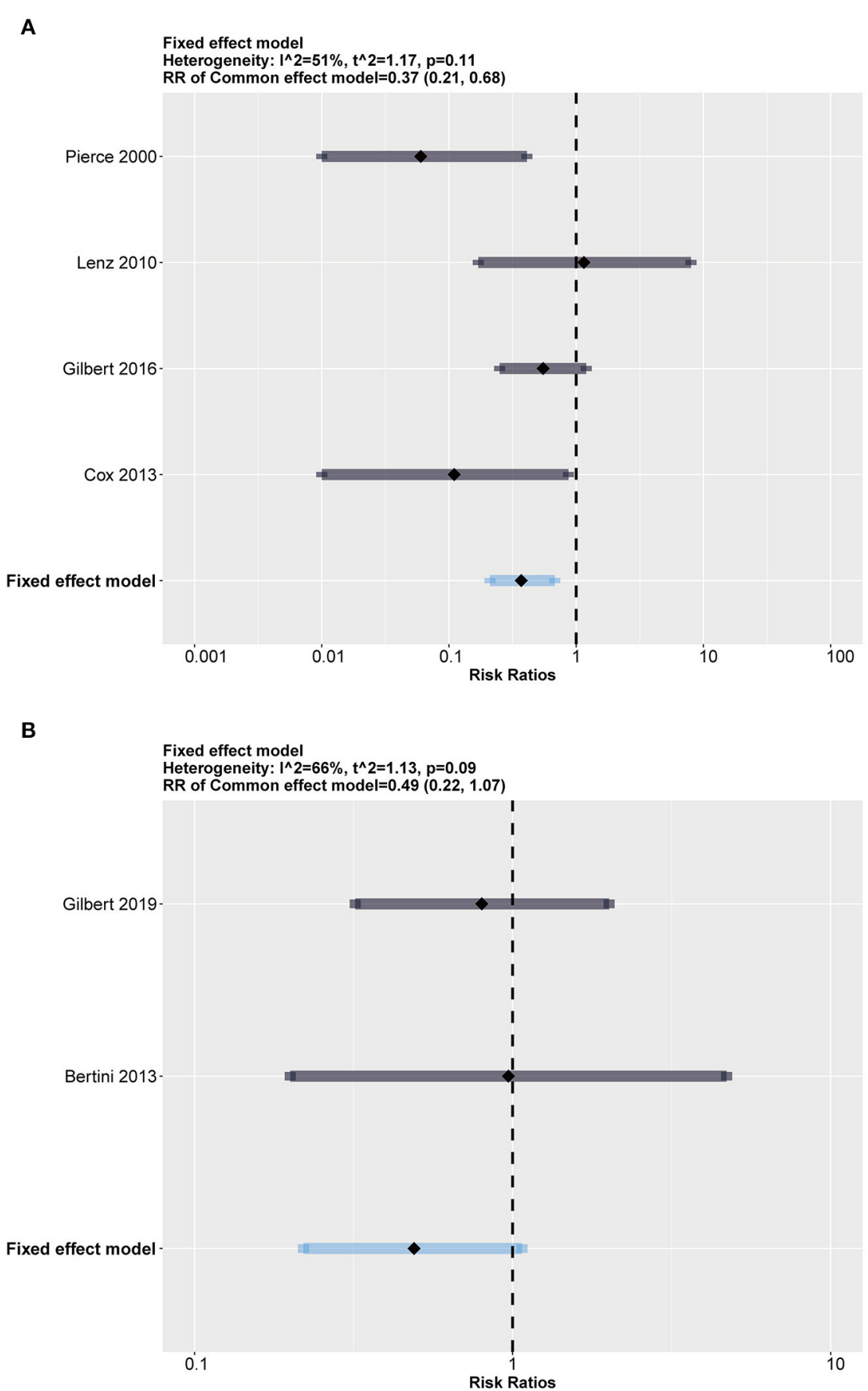

FIGURE 5 | Subgroup analysis between neonates (age $\leq 28$ days) and children (28 days < age $\leq 18$ years). Both groups show high heterogeneity. (A) Forest plot in children (28 days < age $\leq 18$ years) group (compared with standard). (B) Forest plot in neonates (age $\leq 28$ days) group (compared with standard).

change and the duration of catheterization. We attempted to conduct subgroup analysis according to the frequency of dressing change, but the data among the included RCTs were not fully available. Finally, due to data limitations, the role of heparin-impregnated CVCs remains unclear, and future studies evaluating the efficiency of heparin-impregnated CVCs for preventing CRBSI in pediatric patients are warranted.

\section{CONCLUSION}

Our results indicate that antimicrobial-impregnated CVCs have a beneficial effect on decreasing the CRBSI risk in children aged under 18 years compared with non-impregnated standard CVCs, especially in children (28 days $<$ age $\leq$ 18 years). 


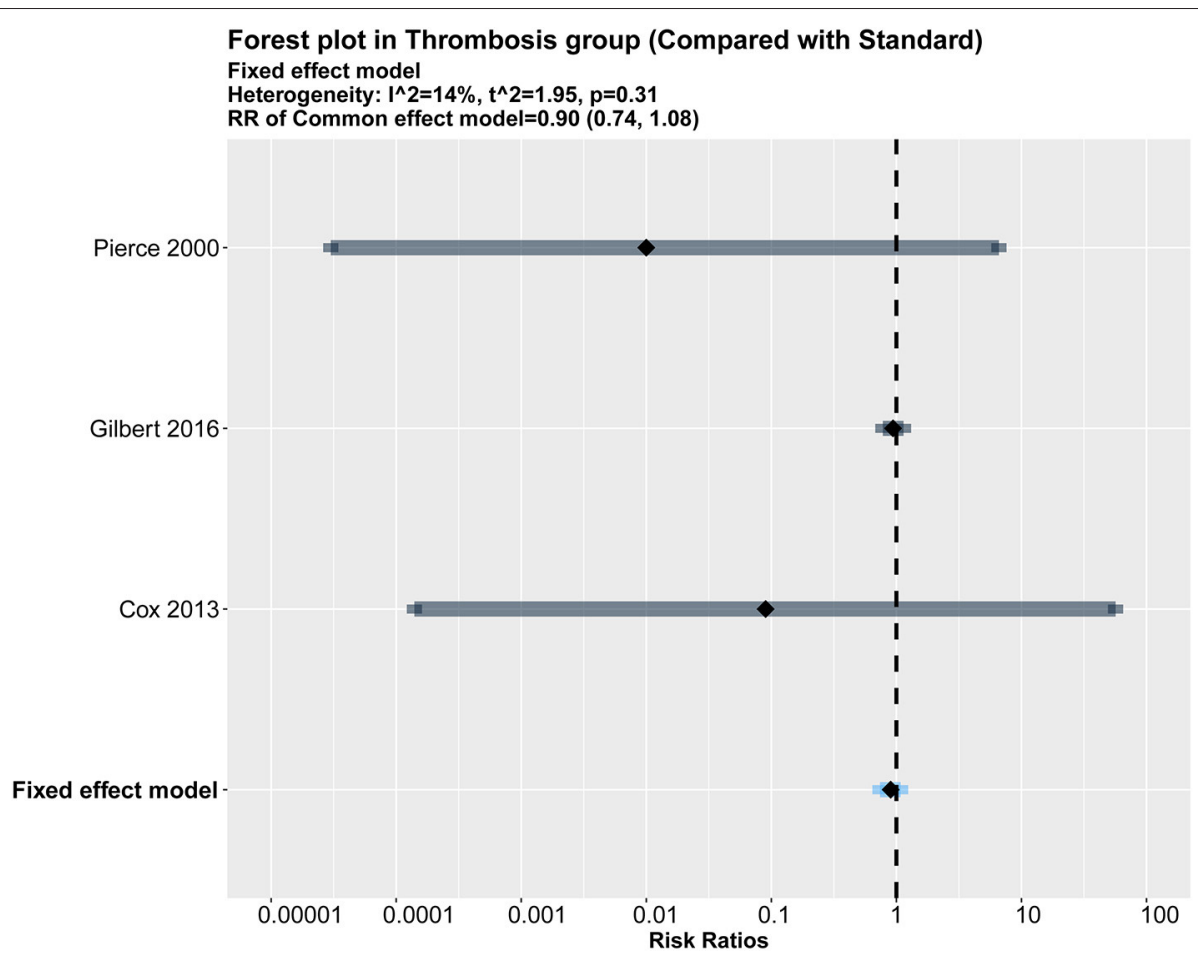

FIGURE 6 | Forest plot of the risk of thrombosis for impregnated catheters.

\section{DATA AVAILABILITY STATEMENT}

The datasets generated for this study are available on request to the corresponding author.

\section{AUTHOR CONTRIBUTIONS}

YM, ZD, and JQ: contributed to the study conception, design, data acquisition, and interpretation.YM, ZD, JQ, HS, and FX: contributed to the data analysis and drafted the manuscript. All authors contributed to the critical revision of the manuscript and approved the final version.

\section{FUNDING}

This study was supported by a grant from the West China Second University Hospital of Sichuan University, KX144,

\section{REFERENCES}

1. Saugel B, Scheeren TWL, Teboul JL. Ultrasound-guided central venous catheter placement: a structured review and recommendations for clinical practice. Crit Care. (2017) 21:225. doi: 10.1186/s13054-017-1814-y

2. Ares G, Hunter CJ. Central venous access in children: indications, devices, and risks. Curr Opin Pediatr. (2017) 29:340-6. doi: 10.1097/MOP.0000000000000485

3. O'Grady NP, Alexander M, Dellinger EP, Gerberding JL, Heard SO, Maki DG, et al. Guidelines for the prevention of intravascular which funded the design of the study and collection, analysis, and interpretation of data and the writing of the manuscript.

\section{SUPPLEMENTARY MATERIAL}

The Supplementary Material for this article can be found online at: https://www.frontiersin.org/articles/10.3389/fped. 2022.795019/full\#supplementary-material

Supplementary Table S1 | Characteristics of the included studies. CICU, cardiac intensive care unit; PICC, peripheral inserted central venous catheter.

Supplementary Table S2 | Details of catheter use in six included studies. We collected detailed information on the catheters used in each study. Sizes differed in accordance with children's age, weight, and health state, as well for lumen numbers. Materials were all the same among six studies after scrutinizing manufacturers of catheters. 
6. Fanaroff AA, Korones SB, Wright LL, Verter J, Poland RL, Bauer CR, et al. Incidence, presenting features risk factors and significance of late onset septicemia in very low birth weight infants. Pediatr Infect Dis J. (1998) 17:593-8. doi: 10.1097/00006454-199807000-00004

7. Lavallee JF, Gray TA, Dumville J, Russell W, Cullum N. The effects of care bundles on patient outcomes: a systematic review and meta-analysis. Implement Sci. (2017) 12:142. doi: 10.1186/s13012-017-0670-0

8. Hu KK, Lipsky BA, Veenstra DL, Saint S. Using maximal sterile barriers to prevent central venous catheter-related infection: a systematic evidence-based review. Am J Infect Control. (2004) 32:142-6. doi: 10.1016/j.ajic.2003.10.006

9. O'Grady NP, Alexander M, Burns LA, Dellinger EP, Garland J, Heard SO, et al. Guidelines for the prevention of intravascular catheter-related infections. Am J Infect Control. (2011) 39:S1-S34. doi: 10.1016/j.ajic.2011.01.003

10. Loveday HP, Wilson JA, Pratt RJ, Golsorkhi M, Tingle A, Bak A, et al. epic3: national evidence-based guidelines for preventing healthcare-associated infections in NHS hospitals in England. J Hosp Infect. (2014) 86:S1-S70. doi: 10.1016/S0195-6701(13)60012-2

11. Chong HY, Lai NM, Apisarnthanarak A, Chaiyakunapruk N. Comparative efficacy of antimicrobial central venous catheters in reducing catheterrelated bloodstream infections in adults: abridged cochrane systematic review and network meta-analysis. Clin Infect Dis. (2017) 64:S131-S40. doi: $10.1093 / \mathrm{cid} / \mathrm{cix} 019$

12. Wei L, Li Y, Li XY, Bian LZ, Wen ZJ, Li M. Chlorhexidine-impregnated dressing for the prophylaxis of central venous catheter-related complications: a systematic review and meta-analysis. Bmc Infect Dis. (2019) 19:429. doi: 10.1186/s12879-019-4029-9

13. Wang HL, Tong HS, Liu HT, Wang Y, Wang R, Gao H, et al. Effectiveness of antimicrobial-coated central venous catheters for preventing catheterrelated blood-stream infections with the implementation of bundles: a systematic review and network meta-analysis. Ann Intensive Care. (2018) 8:71. doi: 10.1186/s13613-018-0416-4

14. Wang H, Huang T, Jing J, Jin J, Wang P, Yang M, et al. Effectiveness of different central venous catheters for catheter-related infections: a network meta-analysis. J Hosp Infect. (2010) 76:1-11. doi: 10.1016/j.jhin.2010.04.025

15. Moher D, Liberati A, Tetzlaff J, Altman DG, Grp P. Preferred reporting items for systematic reviews and meta-analyses: the PRISMA statement. PLoS Med. (2009) 6:e1000097. doi: 10.1371/journal.pmed.1000097

16. Pierce CM, Wade A, Mok Q. Heparin-bonded central venous lines reduce thrombotic and infective complications in critically ill children. Intens Care Med. (2000) 26:967-72. doi: 10.1007/s001340051289

17. Lenz AM, Vassallo JC, Moreno GE, Althabe M, Gómez S, Magliola R, et al. Prevention of catheter-related infection; usefulness and cost-effectiveness of antiseptic catheters in children. Arch Argent Pediatr. (2010) 108:209-15. doi: 10.1590/S0325-00752010000300006

18. Bertini G, Elia S, Ceciarini F, Dani C. Reduction of catheter-related bloodstream infections in preterm infants by the use of catheters with the AgION antimicrobial system. Early Hum Dev. (2013) 89:21-5. doi: 10.1016/j.earlhumdev.2012.07.003

19. Cox EG, Knoderer CA, Jennings A, Brown JW, Rodefeld MD, Walker SG, et al. A randomized, controlled trial of catheter-related infectious event rates using antibiotic-impregnated catheters versus conventional catheters in pediatric cardiovascular surgery patients. J Pediatric Infect Dis Soc. (2013) 2:67-70. doi: $10.1093 /$ jpids/pis066
20. Gilbert RE, Mok Q, Dwan K, Harron K, Moitt T, Millar M, et al. Impregnated central venous catheters for prevention of bloodstream infection in children (the CATCH trial): a randomised controlled trial. Lancet. (2016) 387:1732-42. doi: 10.1016/S0140-6736(16)00340-8

21. Gilbert R, Brown M, Rainford N, Donohue C, Fraser C, Sinha A, et al. Antimicrobial-impregnated central venous catheters for prevention of neonatal bloodstream infection (PREVAIL): an open-label, parallel-group, pragmatic, randomised controlled trial. Lancet Child Adolesc. (2019) 3:381-90. doi: 10.1016/S2352-4642(19)30114-2

22. Mulloy B, Hogwood J, Gray E, Lever R, Page CP. Pharmacology of heparin and related drugs. Pharmacol Rev. (2016) 68:76-141. doi: 10.1124/pr.115.011247

23. Loertzer H, Soukup J, Hamza A, Wicht A, Rettkowski O, Koch E, et al. Use of catheters with the AgION antimicrobial system in kidney transplant recipients to reduce infection risk. Transpl P. (2006) 38:707-10. doi: 10.1016/j.transproceed.2006.01.064

24. Raad I, Darouiche R, Dupuis J, Abi-Said D, Gabrielli A, Hachem R, et al. Central venous catheters coated with minocycline and rifampin for the prevention of catheter-related colonization and bloodstream infections a randomized, double-blind trial. Ann Intern Med. (1997) 127:267-74. doi: 10.7326/0003-4819-127-4-199708150-00002

25. Abou Elella R, Najm HK, Balkhy H, Bullard L, Kabbani MS. Impact of Bloodstream Infection on the Outcome of Children Undergoing Cardiac Surgery. Pediatr Cardiol. (2010) 31:483-9. doi: 10.1007/s00246-009-9624-x

26. Elward AM, Hollenbeak CS, Warren DK, Fraser VJ. Attributable cost of nosocomial primary bloodstream infection in pediatric intensive care unit patients. Pediatrics. (2005) 115:868-72. doi: 10.1542/peds.2004-0256

27. Huang EY, Chen C, Abdullah F, Aspelund G, Barnhart DC, Calkins $\mathrm{CM}$, et al. Strategies for the prevention of central venous catheter infections: an American Pediatric Surgical Association Outcomes and Clinical Trials Committee systematic review. J Pediatr Surg. (2011) 46:2000-11. doi: 10.1016/j.jpedsurg.2011.06.017

28. Wu GS, Chen ZL, Sun Y, Xiao SC, Xia ZF. Impregnated central venous catheters in children: a systematic review of randomized controlled trials. Intens Care Med. (2017) 43:1159-61. doi: 10.1007/s00134-017-4777-1

Conflict of Interest: The authors declare that the research was conducted in the absence of any commercial or financial relationships that could be construed as a potential conflict of interest.

Publisher's Note: All claims expressed in this article are solely those of the authors and do not necessarily represent those of their affiliated organizations, or those of the publisher, the editors and the reviewers. Any product that may be evaluated in this article, or claim that may be made by its manufacturer, is not guaranteed or endorsed by the publisher.

Copyright (c) 2022 Deng, Qin, Sun, Xv and Ma. This is an open-access article distributed under the terms of the Creative Commons Attribution License (CC BY). The use, distribution or reproduction in other forums is permitted, provided the original author(s) and the copyright owner(s) are credited and that the original publication in this journal is cited, in accordance with accepted academic practice. No use, distribution or reproduction is permitted which does not comply with these terms. 